\title{
The impact of the undetected COVID-19 cases on its transmission dynamics
}

\author{
Sujata Saha $\mathbb{0} \cdot$ Sumanta Saha
}

Received: 16 June 2020/Accepted: 24 November 2020/Published online: 14 June 2021

(C) The Indian National Science Academy 2021

\begin{abstract}
Objective The COVID-19 pandemic is currently ongoing. Presently, due to the unavailability of a definitive vaccine to decrease its acquiring, it's essential to understand its transmissibility in the community by undetected cases to control its transmission. This study aims to study this context using mathematical modelling.

Methods A COVID-19 transmission model was framed that estimated the basic reproduction number $R_{0}$ (a measurement of disease risk) using the next-generation method. It explored the contribution of exposed and infected (detected and undetected) individuals, and environmental pathogen to the overall risk of infection spreading, utilizing the publicly reported data of this infection in Maharashtra between March 22, 2020, and May 4, 2020. A sensitivity analysis was performed to study the effect of a rising number of undetected cases to $R_{0}$.

Results The estimated basic reproduction number is $\boldsymbol{R}_{0}=4.63$, which increases rapidly with the rise in the undetected COVID-19 cases. Although the exposed individuals made the largest contribution to infection transmission $\left(\boldsymbol{R}_{1}=2.42\right)$, the contaminated environment also played a significant role.

Conclusions It is crucial to identify the individuals exposed and infected to COVID-19 disease and isolate them to control its transmission. The awareness of the role of fomites in infection transmission is also important in this regard.
\end{abstract}

Keywords Mathematical model · Coronavirus · Pandemics · Disease transmission - infectious · Basic reproduction number $\cdot$ Infectious disease outbreaks $\cdot$ India

\section{Introduction}

World Health Organization (WHO) declared the ongoing coronavirus disease 2019 (COVID-19) epidemic as a Global Public Health Emergency of International Concern on January 30, 2020 [1]. COVID-19 started from China in December 2019 [2, 3], which is caused by Severe Acute Respiratory Syndrome Coronavirus-2 (SARS-CoV-2), an enveloped single-stranded RNA virus of zoonotic origin $[1,3]$.

Communicated by G.D. Veerappa Gowda.

S. Saha $(\bowtie)$

Mankar College, Mankar, India

E-mail: sahasujata@outlook.com

S. Saha

R. G. Kar Medical College, Kolkata, West Bengal 700004, India 
India has 182143 number of active COVID-19 cases and 5164 number of deaths as of May 31, 2020. Since there is no effective therapeutics or licensed vaccine to combat novel COVID-19 [1,4], contact tracing and quarantining [5] is one of the major strategies adopted by India to control its transmission and mortality. But, while interviewing the COVID-19 infected individuals for contact tracing, some contacts may be missed due to recall bias. Such missed COVID-19 infected individuals who remain asymptomatic during its incubation period (2-14 days), increases the risk of community transmission unknowingly [1].Therefore, it is crucial to study their role in disease transmission.

Several, research papers have tried to understand the transmission dynamics of this disease using mathematical modelling. Choi \& Ki [2] framed a susceptible-exposed-infected-hospitalized-recovered (SEIHR) model and calculated the basic reproduction number as 4.028 (95\% CI: 4.010,4.046) using the reported number of confirmed cases from January 20, 2020, and March 4, 2020, in Korea. Yang \& Wang [1] modelled considering the pathogen concentration in the environmental reservoir and its role in disease transmission. Mandal et al. [4] framed an SEIR (susceptible-exposed-infected-recovered) model with an objective to prevent, or delay, the local outbreaks in India through restrictions on travel from COVID-19 affected countries. Read et al. [6] reported the basic reproduction number as 3.11 by fitting data of a deterministic SEIR metapopulation transmission model. As these studies primarily studied the disease risk via direct human-to-human transmission route, the role of undetected cases on the risk of infection spread in the community remains unclear.

Therefore, in this study we aimed to study this under-explored area of COVID-19 research by mathematical modelling, assuming the disease acquiring potential of susceptible individuals with contact to this virus-infected asymptomatic and symptomatic individuals, and virus-contaminated fomites.

\section{Notations}

We used the following notations to develop the model -

$S$ Susceptible individuals

$E$ Exposed persons

$I_{1}$ Infected people, who are detected through proper testing

$I_{2}$ Infected individuals who are undetected

$R$ Recovered population

$V$ environmental reservoir of the pathogen (i.e. coronavirus-contaminated fomites)

$\beta_{e}$ Transmission rates between the exposed and susceptible individuals

$\beta_{i 1}$ Transmission rates between the detected infected and susceptible individuals

$\beta_{i 2}$ Transmission rates between the undetected infected and susceptible individuals

$\beta_{v}$ Environment to human transmission rates

$\pi$ Influx rate in the population

$\mu$ Natural death rate in the population

$\gamma$ The disease recovery rate

$w$ Disease induced death rate

$\alpha^{-1}$ Incubation period

$\beta$ Percentage of the infected individuals who are undetected

$\xi_{1}$ Contribution of the exposed individuals to the environmental reservoir of the pathogen (i.e. coronavirus-contaminated fomites)

$\xi_{2}$ Contribution of the detected infected individuals to the environmental reservoir of the pathogen (i.e. coronavirus-contaminated fomites)

$\xi_{3}$ Contribution of the undetected infected individuals to the environmental reservoir of the pathogen (i.e. coronavirus-contaminated fomites)

$\sigma$ Removal rate of the coronavirus from the environment

$F$ Infection matrix

$T$ Transition matrix

$K$ Next-generation matrix

$\rho$ Spectral radius of the next generation matrix 


\section{Mathematical methods}

In this model, we divided the total human population into five compartments - susceptible (S), exposed (E), infected detected $\left(I_{1}\right)$, infected undetected $\left(I_{2}\right)$, and recovered $(\mathrm{R})$. Here, we have introduced another compartment $(\mathrm{V})$ for the environmental reservoir of the coronavirus pathogen that contributes to spread the disease. Individuals in the exposed class are asymptomatic infected, but they are capable to spread the disease. People in both the infected classes (detected and undetected) are fully symptomatic, but $I_{1}$ consists those who are detected by proper testing system and are isolated, whereas, $I_{2}$ represents the undetected cases who are living in the society as usual. An additional compartment has been introduced for the environmental reservoir of this novel pathogen. The following differentials equations describe the transmission dynamics of the disease COVID-19 -

$$
\begin{gathered}
\frac{d S}{d t}=\pi-\beta_{e} S E-\beta_{i 1} S I_{1}-\beta_{i 2} S I_{2}-\beta_{v} S V-\mu S \\
\frac{d E}{d t}=\beta_{e} S E+\beta_{i 1} S I_{1}+\beta_{i 2} S I_{2}+\beta_{v} S V-(\alpha+\mu) E \\
\frac{d I_{1}}{d t}=\alpha \beta E-(\gamma+\mu+w) I_{1} \\
\frac{d I_{2}}{d t}=\alpha(1-\beta) E-(\gamma+\mu+w) I_{1} \\
\frac{d R}{d t}=\gamma\left(I_{1}+I_{2}\right)-\mu R \\
\frac{d V}{d t}=\xi_{1} E+\xi_{2} I_{1}+\xi_{3} I_{2}-\sigma V
\end{gathered}
$$

Clearly, the system has a disease-free equilibrium at $\left(\frac{\pi}{\mu}, 0,0,0,0,0\right)$.

We will calculate the basic reproduction number $\mathrm{R}_{0}$ using the next-generation approach. In this approach, like offspring production, giving birth to a new infected individual is regarded as the infection transmission [7], and the growth factor per generation is characterized by the basic reproduction number $\mathrm{R}_{0}$. Here, we introduce a matrix, called the next-generation matrix, that relates the number of newly infected individuals in the various compartments in consecutive generations and $\mathrm{R}_{0}$ is defined as the spectral radius of this matrix [7].

In this paper, we derive the next-generation matrix using Van den Driessche and Watmough Approach. According to this approach, we will divide the compartments into infected and non-infected compartments. Now, if $\mathrm{x}$ be the vector of dependent variables in the infected compartments, and $\mathrm{y}$ be the vector of variables in the noninfected compartments i.e. $\mathrm{x}=\left(\mathrm{x}_{1}, \mathrm{x}_{2}, \mathrm{x}_{3}, \mathrm{x}_{4}\right)$ and $\mathrm{y}=\left(\mathrm{y}_{1}, \mathrm{y}_{2}\right)$ where, $x_{i}(\mathrm{i}=1,2,3,4)$ represent the infection components $E, I_{1}, I_{2}$, and $V$ respectively and $y_{j}(\mathrm{j}=1,2)$ represent the non-infection compartment $\mathrm{S}$ and $\mathrm{R}$ respectively, the above system of equations (1) can be written as

$$
\begin{gathered}
x_{i}^{\prime}=f_{i}(x, y) \\
y_{j}^{\prime}=g_{j}(x, y)
\end{gathered}
$$

where,

$$
\begin{gathered}
f_{1}(x, y)=\beta_{e} S E+\beta_{i 1} S I_{1}+\beta_{i 2} S I_{2}+\beta_{v} S V-(\alpha+\mu) E \\
f_{2}(x, y)=\alpha \beta E-(\gamma+\mu+w) I_{1} \\
f_{3}(x, y)=\alpha(1-\beta) E-(\gamma+\mu+w) I_{2} \\
f_{4}(x, y)=\xi_{1} E+\xi_{2} I_{1}+\xi_{3} I_{2}-\sigma V
\end{gathered}
$$

and,

$$
\begin{gathered}
g_{1}(x, y)=\varpi-\beta_{e} S E-\beta_{i 1} S I_{1}-\beta_{i 2} S I_{2}-\beta_{v} S V-\mu S \\
g_{2}(x, y)=\gamma\left(I_{1}+I_{2}\right)-\mu R
\end{gathered}
$$

Next, we split the right-hand side in the infection compartment in the following way 


$$
\begin{array}{r}
x_{i}^{\prime}=P_{i}(x, y)-Q_{i}(x, y) \\
y_{j}^{\prime}=g_{j}(x, y)
\end{array}
$$

where, $P_{i}(x, y)$ represent the rate of appearance of new infection in the compartment $\mathrm{x}_{\mathrm{i}}$ ( for $\left.\mathrm{i}=1,2,3,4\right)$ and $Q_{i}(x, y)$ represent the remaining transitional terms, namely births, deaths, recovery, and change in infection status (such as exposed to infected etc.). Therefore,

$$
\begin{array}{ll}
P_{1}(x, y)=\beta_{e} S E+\beta_{i 1} S I_{1}+\beta_{i 2} S I_{2}+\beta_{v} S V, & Q_{1}(x, y)=(\alpha+\mu) E \\
P_{2}(x, y)=0, & Q_{2}(x, y)=-\alpha \beta E+(\gamma+\mu+w) I_{1} \\
P_{3}(x, y)=0, & Q_{3}(x, y)=-\alpha(1-\beta) E+(\gamma+\mu+w) I_{2} \\
P_{4}(x, y)=0, & Q_{4}(x, y)=-\xi_{1} E-\xi_{2} I_{1}-\xi_{3} I_{2} \mp \sigma V
\end{array}
$$

For the infected compartments, the linearized system can be written as -

$$
x_{i}^{\prime}=(F-T) x
$$

Here, $\mathrm{F}$ and $\mathrm{T}$ are the infection matrix and the transition matrix, defined by -

$$
F=\left[\frac{\partial P_{i}}{\partial x_{j}}\right], T=\left[\frac{\partial Q_{i}}{\partial x_{j}}\right]
$$

which appear from the linearization of the system, around the disease-free equilibrium. Hence,

$$
F=\left[\begin{array}{cccc}
\beta_{e} S_{0} & \beta_{i 1} S_{0} & \beta_{i 2} S_{0} & \beta_{v} S_{0} \\
0 & 0 & 0 & 0 \\
0 & 0 & 0 & 0 \\
0 & 0 & 0 & 0
\end{array}\right], T=\left[\begin{array}{cccc}
\alpha+\mu & 0 & 0 & 0 \\
-\alpha \beta & w_{1} & 0 & 0 \\
-\alpha(1-\beta) & 0 & w_{1} & 0 \\
-\xi_{1} & -\xi_{2} & -\xi_{3} & \sigma
\end{array}\right]
$$

where,

$$
S_{0}=\frac{\pi}{\mu}, \text { and } w_{1}=(\gamma+\mu+w)
$$

The next-generation matrix is defined as $K=F T^{-1}$.

The basic reproduction number $R_{0}$ of the model (1), is defined by the spectral radius of the nextgeneration matrix $K$, therefore,

$$
R_{0}=\rho\left(F T^{-1}\right)=\frac{\beta_{e} S_{0}}{(\alpha+\mu)}+\frac{\alpha \beta \beta_{i 1} S_{0}}{(\alpha+\mu) w_{1}}+\frac{\alpha(1-\beta) \beta_{i 2} S_{0}}{(\alpha+\mu) w_{1}}+\frac{\left\{\alpha \beta \xi_{2} w_{1}+\left(\xi_{1} w_{1}+\alpha(1-\beta) \xi_{3} w_{1}\right)\right\} \beta_{v} S_{0}}{(\alpha+\mu) w_{1}^{2} \sigma}
$$

Or,

$$
R_{0}=R_{1}+R_{2}+R_{3}+R_{4}
$$

where, $R_{1}, R_{2}, R_{3}$ and $R_{4}$ gives the quantification of the disease risk through susceptible-to-exposed, susceptible-to-infected detected, susceptible-to-infected undetected, and environment-to-human transmission routes, respectively.

\section{Numerical results}

Here, this model is applied to estimate the basic reproduction number using the COVID-19 outbreak data published by WHO and the Ministry of Health and Family Welfare, India for the epidemic period between March 22 and May 4, 2020 for the Indian state of Maharashtra [8-10]. The data provides the daily confirm cases, recoveries, and the total death caused by COVID- 19 .

Since the state was in complete lockdown during this period, its population influx rate was expected to be equal to its birth rate, which was estimated at 5603/day [11]. The total population of Maharashtra (N) is 112374333 [12]. As of 22-March 2020, the total confirmed coronavirus cases were 69 including 2 death and 0 recoveries, but these counts rose to 14541, 583, and 2465 respectively on 4th May 2020 [9, 10]. Therefore, $S(0)=112374264$. Again, as both the exposed and undetected infected persons tend to live in the society, as 
Table 1 The value of the parameters associated with this model

\begin{tabular}{lll}
\hline Parameter & Estimated values & Sources \\
\hline$\beta_{e}$ & $0.25 \times 0.1231 \times 10^{-7}$ & {$[13]$} \\
$\beta_{i 1}$ & $0.25 \times 0.5944 \times 0.1231 \times 10^{-7}$ & {$[13]$} \\
$\beta_{i 2}$ & $0.25 \times 0.1231 \times 10^{-7}$ & {$[13]$} \\
$\beta_{v}$ & $1.03 \times 10^{-8}$ & {$[1]$} \\
$\pi$ & $5603 /$ day & $[1]]$ \\
$\mu$ & $4.99 \times 10^{-5} /$ day & {$[11]$} \\
$\gamma$ & 0.1695 & Estimated \\
$w$ & 0.04 & Estimated \\
$\alpha^{-1}$ & $1 / 7$ & {$[1]$} \\
$\beta$ & 0.9 & Assumed \\
$\xi_{1}$ & 0.001 & {$[13]$} \\
$\xi_{2}$ & 0.000398 & {$[13]$} \\
$\xi_{3}$ & 0.001 & {$[13]$} \\
\hline
\end{tabular}

Table 2 Impact of undetected cases on COVID-19 disease risk

\begin{tabular}{llllll}
\hline Number of undetected cases $(1-\beta)$ & $R_{0}$ & $R_{1}$ & $R_{2}$ & $R_{3}$ & $R_{4}$ \\
\hline 0.1 & 4.63 & 2.42 & 1.73 & 0.323 & 0.161 \\
0.2 & 4.759 & 2.416 & 1.535 & 0.645 & 0.162 \\
0.3 & 4.891 & 2.416 & 1.343 & 0.968 & 0.164 \\
0.4 & 5.023 & 2.416 & 1.151 & 1.291 & 0.165 \\
\hline
\end{tabular}

usual, they transmit the disease at the same rate, the values of these two parameters $\beta_{e}$ and $\beta_{i 2}$ were taken as equal to $0.25 \times 0.1231 \times 10^{-7}$ (according to Shaikh et al., 2020) [13]. Also, the estimated recovery rate $(\gamma)$ extracted from the data is 0.012 per day. Moreover, the COVID-19 affected family members can survive in the environment from a few hours to several days [1], we considered this value $5 \mathrm{~h}$ in this study and therefore, the virus removal rate $(\sigma)$ is $0.2 /$ day. We provided the value of the parameters and its sources in Table 1.

Using the above-mentioned values of the parameters, the basic reproduction number was estimated as $R_{0}=4.63$, with $R_{1}=2.42, R_{2}=1.73, R_{3}=0.323$, and $R_{4}=0.161$, establishing the highest contribution of exposed-to-susceptible transmission route to the disease risk.

Next, regarding the impact of undetected cases on the disease risk, Table 2 shows a sharp increase in the disease risk with a rise in this number.

\section{Discussion}

This study found a significant contribution of undetected COVID-19 cases to the transmission risk of the disease $\left(R_{3}=0.323\right)$. The sensitivity analysis (provided in Table 2$)$ showed a rapid increase in the disease risk with the rise in this factor. Also, the highest rate of infection spreading is caused by the exposed individuals $\left(\left(R_{1}=2.42\right)\right)$, establishing the fact that as the exposed individuals remain asymptomatic in the incubation period of 2-14 days, they can easily transmit the disease unknowingly to the other people in their surroundings. Furthermore, the contaminated environment also contributes to the overall disease risk.

Next, the conceivable implications of this study are specified here. At the current time, when the pandemic claims billions of lives globally with no signs of slowing down due to non-availability of vaccine or definitive treatment, the findings of this study may help in detecting better preventive health policy initiatives in terms of decreasing the speed of social transmission. In this regard, the presently proposed actions like social distancing and the implementation of lockdown appear to be justified to curb the pace of disease transmission. Besides, this model hints, the environmental components like fomites might have some role in disease transmission, therefore, the use of personal protective equipment, like masks, gloves, hand sanitizer seems to be essential.

Regarding the strengths of this paper, this is perhaps the first study on COVID-19 that explored the role of positive non-detected cases on disease transmission in the community, using a mathematical model. Furthermore, the use of recent outbreak data makes the results of this study relevant to the current pandemic 
scenario. Also, the inclusion of the environment as a component of the mathematical model helps to understand the agent, host, and environment relationship of COVID-19 better.

Despite these strengths, this study has a few weaknesses. Since, the basic reproduction number was estimated by taking some parameter values from other studies and an approximated value of the number of undetected cases, the findings of this model may differ slightly from its actual value.

\section{Conclusions}

The COVID-19 disease risk increases with the growing number of undetected cases. Besides, individuals exposed to SARS-CoV-2 infected individuals can unknowingly spread the infection at a very high rate to those around them. Therefore, to control the disease spread, it is crucial to identify and socially isolate such undetected and exposed cases.

Authors contribution The concept, design, analysis, and the manuscript draft were primarily prepared by $\mathrm{SS}^{1} . \mathrm{SS}^{2}$ aided in conception, interpretation and hard editing of the manuscript.

Funding No funding received for this study.

\section{Declarations}

Conflict of interest None declared.

Ethical clearance An ethical clearance was not required.

\section{References}

1. Yang C, Wang J. A mathematical model for the novel coronavirus epidemic in Wuhan, China. Math Biosci Eng [Internet]. 2020;17(3):2708-24. Available from: http://www.aimspress.com/article/ https://doi.org/10.3934/mbe.2020148

2. Choi SC, Ki M. Estimating the reproductive number and the outbreak size of Novel Coronavirus disease (COVID-19) using mathematical model in Republic of Korea. Epidemiol Health [Internet]. 2020 Mar 12;42:e2020011. Available from: http://e-epih.org/journal/view.php? https://doi.org/10.4178/epih.e2020011

3. Oren O, Kopecky SL, Gluckman TJ, Gersh BJ, Blumenthal RS. Coronavirus Disease 2019 (COVID-19): Epidemiology, Clinical Spectrum and Implications for the Cardiovascular Clinician [Internet]. AMERICAN COLLEGE of CARDIOLOGY. 2020 [cited 2020 May 12]. p. Expert Analysis. Available from: https://www.acc.org/latest-in-cardiology/articles/ 2020/04/06/11/08/covid-19-epidemiology-clinical-spectrum-and-implications-for-the-cv-clinician

4. Mandal S, Bhatnagar T, Arinaminpathy N, Agarwal A, Chowdhury A, Murhekar M, et al. Prudent public health intervention strategies to control the coronavirus disease 2019 transmission in India: A mathematical model-based approach. Indian J Med Res [Internet]. 2020; Available from: http://www.ijmr.org.in/preprintarticle.asp?id=281325;type $=0$

5. Vaidyanathan G. People power: How India is attempting to slow the coronavirus. Nature. 2020 Apr 10

6. Intergovernmental Panel on Climate Change. Summary for Policymakers. In: Climate Change 2013 - The Physical Science Basis: Working Group I Contribution to the Fifth Assessment Report of the Intergovernmental Panel on Climate Change. In: Intergovernmental Panel on Climate Change, editor. Climate Change 2013 - The Physical Science Basis [Internet]. Cambridge: Cambridge University Press; 2020. p. 1-30. Available from: https://www.cambridge.org/core/ product/identifier/CBO9781107415324A009/type/book_part

7. Martcheva M. An Introduction to Mathematical Epidemiology [Internet]. Boston, MA: Springer US; 2015.106 p. (Texts in Applied Mathematics; vol. 61). Available from: http://link.springer.com/ https://doi.org/10.1007/978-1-4899-7612-3

8. WHO. Novel Coronavirus Disease (COVID-19) Situation Update Report - 8 [Internet]. World Health Organization, India. 2020 [cited $2020 \mathrm{Apr}$ 28]. Available from: https://www.who.int/docs/default-source/wrindia/situation-report/indiasituation-report-8.pdf?sfvrsn=cd671813_2

9. Wikipedia the free encyclopedia. COVID-19 pandemic in Maharashtra [Internet]. 2020 [cited 2020 May 30]. Available from: https://en.wikipedia.org/wiki/COVID-19_pandemic_in_Maharashtra\#May

10. COVID19 Maharashtra Location Cases [Internet]. [cited 2020 May 30]. Available from: https://phdmah.maps.arcgis.com/ apps/opsdashboard/index.html\#/2cc0055832264c5296890745e9ea415c

11. Wikipedia the free encyclopedia. Demographics of India [Internet]. 2020 [cited 2020 May 4]. Available from: https://en. wikipedia.org/wiki/Demographics_of_India

12. Wikipedia the free encyclopedia. 2011 Census of India [Internet]. 2020 [cited 2020 May 4]. Available from: https://en. wikipedia.org/wiki/2011_Census_of_India\#Population

13. Shaikh AS, Shaikh IN, Nisar KS. A Mathematical Model of COVID-19 Using Fractional Derivative: Outbreak in India with Dynamics of Transmission and Control. 2020 Apr 9 